\title{
Single-Session Treatment of Cholecysto-Choledocholithiasis: Totally Laparoscopic versus Laparo-Endoscopic
}

\author{
Mostafa Mohamoud Sayed ${ }^{1}$, Ayman Kamal ${ }^{1}$, Abdallah Mohamed Taha ${ }^{2, *}$, \\ Mahmoud Abdelhameid ${ }^{2}$, Ahmed Mohamed Ali Abdallah ${ }^{1}$, Zein Sayed ${ }^{3}$ \\ ${ }^{1}$ General Surgery Department, Faculty of Medicine, Assuit University, Assiut, Egypt \\ ${ }^{2}$ General Surgery Department, Faculty of Medicine, South Valley University, Qena, Egypt \\ ${ }^{3}$ Internal Medicine Department, Faculty of Medicine, Assuit University, Assiut, Egypt \\ Email address: \\ abdallahsurgery@yahoo.com (A. M. Taha) \\ ${ }^{*}$ Corresponding author
}

\section{To cite this article:}

Mostafa Mohamoud Sayed, Ayman Kamal, Abdallah Mohamed Taha, Mahmoud Abdelhameid, Ahmed Mohamed Ali Abdallah, Zein Sayed. Single-Session Treatment of Cholecysto-Choledocholithiasis: Totally Laparoscopic versus Laparo-Endoscopic. Journal of Surgery. Vol. 5, No. 5, 2017 pp. 72-78. doi: 10.11648/j.js.20170505.11

Received: July 2, 2017; Accepted: July 11, 2017; Published: September 13, 2017

\begin{abstract}
Background: This study details Assiut and South Valley universities experience in treating combined gall bladder and common bile duct stones in a single session, either with Endoscopic Retrograde Cholangio-Pancreatography (ERCP) for Common Bile Duct (CBD) stone extraction followed by laparoscopic cholecystectomy (LC), or totally laparoscopic treatment. Patients and methods: In this prospective randomized study, 46 consecutive patients with confirmed cholecystocholedocholithiasis were randomized to 2 groups. Group (A) included 24 patients treated with single-session ERCP for CBD stone extraction and laparoscopic cholecystectomy [ERCP-LC]. Group (B) included 22 patients treated with laparoscopic CBD exploration and laparoscopic cholecystectomy [LCBDE-LC]. Demographic data, operative time, CBD clearance success rate, short term complications and duration of hospital stay were recorded. Results: Patients included 28 females and 18 males with mean age of $42.1 \pm 12.1$ years (range 17 - 71 years). In 22/24 patients (91.7\%) ERCP-LC was done successfully. Mean operative time was $105 \pm 19.1$ minutes (50-150 min.). No intra-operative complications occurred. Early post-operative complications occurred in 3 patients (12.5\%). Mean hospital stay was 2.1 \pm 0.91 days (1-6 days). In the other group, LCBDELC was performed successfully in 22/22 patients (100\%). Mean operative time was $145 \pm 23$ minutes (100-180 min.). Minor intra-operative complications (bleeding) occurred in $2 / 22$ cases $(9 \%)$. Minor early post-operative complications (bile leak, ileus, bleeding) occurred in $4 / 22$ patients (18\%). Mean hospital stay was $2.8 \pm 0.83$ days (2-7 days). Conclusion: Single session ERCP-LC and LCBDE-LC procedures for management of cholecysto-choledocholithiasis are feasible, safe, and effective and have comparable outcome regarding success rate, peri-operative complications. ERCP-LC has statistically significant less operative time and less hospital stay.
\end{abstract}

Keywords: Common Bile Duct Stones, Common Bile Duct Exploration,

Endoscopic Retrograde Cholangio-Pancreatography, Endoscopic Sphincterotomy, Choledocholithiasis

\section{Introduction}

Approximately, $20 \%$ of symptomatic gall bladder stones are associated with common bile duct stones. Hence, all patients with gall bladder stones should be assessed for the possibility of having $\mathrm{CBD}$ stones by abdominal US and serum bilirubin and alkaline phosphatase levels. When these investigations are not conclusive, then magnetic resonance cholangiopancreatography (MRCP) should be done [1-3]. Common bile duct (CBD) stones should be removed to prevent the potential serious complications as obstructive jaundice, cholangitis, and biliary pancreatitis [4].

Pre-operative ERCP and endoscopic sphincterotomy (ES) 
for CBD stones extraction is a relatively safe and effective option for removing CBD stones in most cases thus, avoiding CBD exploration [5]. Albeit, it necessitates a second procedure and there is a risk that it will be unsuccessful, requiring surgical CBD exploration [6].

There is a debate regarding single session treatment of gall bladder stones with CBD stones, either it should be totally laparoscopic (laparoscopic CBD exploration and stone extraction followed by cholecystectomy) or combines intraoperative ERCP for CBD stones extraction with laparoscopic cholecystectomy LC [7].

Totally laparoscopic single-session procedure was equal to that achieved with pre-operative ERCP and LC but had the advantage of lower morbidity and a shorter hospitalization for the patients [8].

Totally laparoscopic CBD exploration and stone extraction (LCBDE) followed by LC allows for a minimally invasive, single-session, single-technique for both diagnosis (intraoperative cholangiography) and treatment without the drawbacks of ERCP. This technique has an overall CBD clearance of 75-92\% in experienced hands [7].

The aim of this study is to compare the outcome of intraoperative ERCP followed by laparoscopic cholecystectomy (ERCP-LC) versus totally laparoscopic common bile duct exploration and laparoscopic cholecystectomy (LCBDE-LC) as a single session for treatment of cholecystocholedocholithiasis regarding success rate, operative time and perioperative complications.

\section{Patients and Methods}

\subsection{Patients}

This prospective randomized study included 46 patients with confirmed cholecysto-choledocholithiasis by US (ultrasonography) and MRCP (Magnetic Resonance Cholangio-Pancreatography), who were admitted to the Surgery Departments in Assuit University Hospital and South Valley University Hospital, between April 2014 and April 2017. Patients were suitable for either interventions, ERCP-LC or LCBDE-LC.

Exclusion criteria included patients unfit for surgery and patients with confounding factors that affect the outcome are excluded (i.e. previous ERCP, suspected Mirizzi syndrome, intrahepatic bile duct stones, multiple CBD stones $(>10)$, Dilated CBD diameter $>2 \mathrm{~cm}$, or stone size $>2 \mathrm{~cm}$ ), and those with acute cholangitis or pancreatitis or suspected bile duct strictures or malignancy.

\subsection{Preoperative Work-up}

History taking, physical examination, and routine laboratory investigations in the form of complete blood count, liver and kidney function tests, and evaluation of PT and INR were performed for all patients. CBD stones were diagnosed by abdominal US and MRCP.

\subsection{Intervention}

Patients were randomly divided into two groups. In group (A), patients $(n=24)$ were treated with single-session intraoperative ERCP and laparoscopic cholecystectomy [ERCPLC]. In group (B), patients $(n=22)$ were treated with laparoscopic $\mathrm{CBD}$ exploration and stone extraction together with laparoscopic cholecystectomy [LCBDE-LC].

All patients signed an informed consent after explanation of the technique to be performed, other options for treatment, possible complications and the expected success rate. All patients were fasting six hours before the procedure. Prophylactic antibiotic (Ceftriaxone, 1 gm.) was given to all patients at the time of induction of general anesthesia. The IV antibiotics were continued for $48 \mathrm{~h}$ and then switched to oral antibiotics.

\subsubsection{Operative Technique for Group (A)}

In left lateral decubitus, Endoscopic guide-wire assisted cannulation of the CBD was done followed by injection of contrast to delineate the anatomy of the CBD and intrahepatic bile ducts and to confirm the number, size and distribution of the CBD stones. Sphinctrotomy was then done and Common bile duct stone extraction was done with a balloon catheter inflated with saline in most cases. In some cases Dormia basket was used to remove CBD stones when the balloon kept slipping beside the stones. This happened in 6 patients with small stones in relatively wide CBDs (Figure 1).

After finishing the ERCP procedure, suction of air inflated during endoscopy was done and then the patients were carefully turned to supine position and prepared for laparoscopic cholecystectomy. After dissection of the Callot's triangle, during laparoscopic cholecystectomy, a metallic clip was inserted between the cystic duct and the gall bladder neck. Then the cystic duct was cannulated and diluted urographin cholangiography was done to confirm patency of the CBD after the ERCP. This procedure aimed to eliminating the possibility of missed distal cystic duct stones that can pass into CBD during gall bladder and cystic duct manipulation.

\subsubsection{Operative Technique for Group (B) (Figure 2)}

In the supine position, Laparoscopic cholecystectomy was performed using the standard four trocar technique. The Calot's triangle was dissected and cystic artery identified, clipped and cut. Then, the cystic duct was clipped close to the gall bladder neck and a small incision performed near the clip for introduction of a 6 Fr. ureteric catheter. Diluted urographin was then injected into the catheter and intraoperative cholangiography was performed using a C-arm Xray to confirm MRCP findings. 

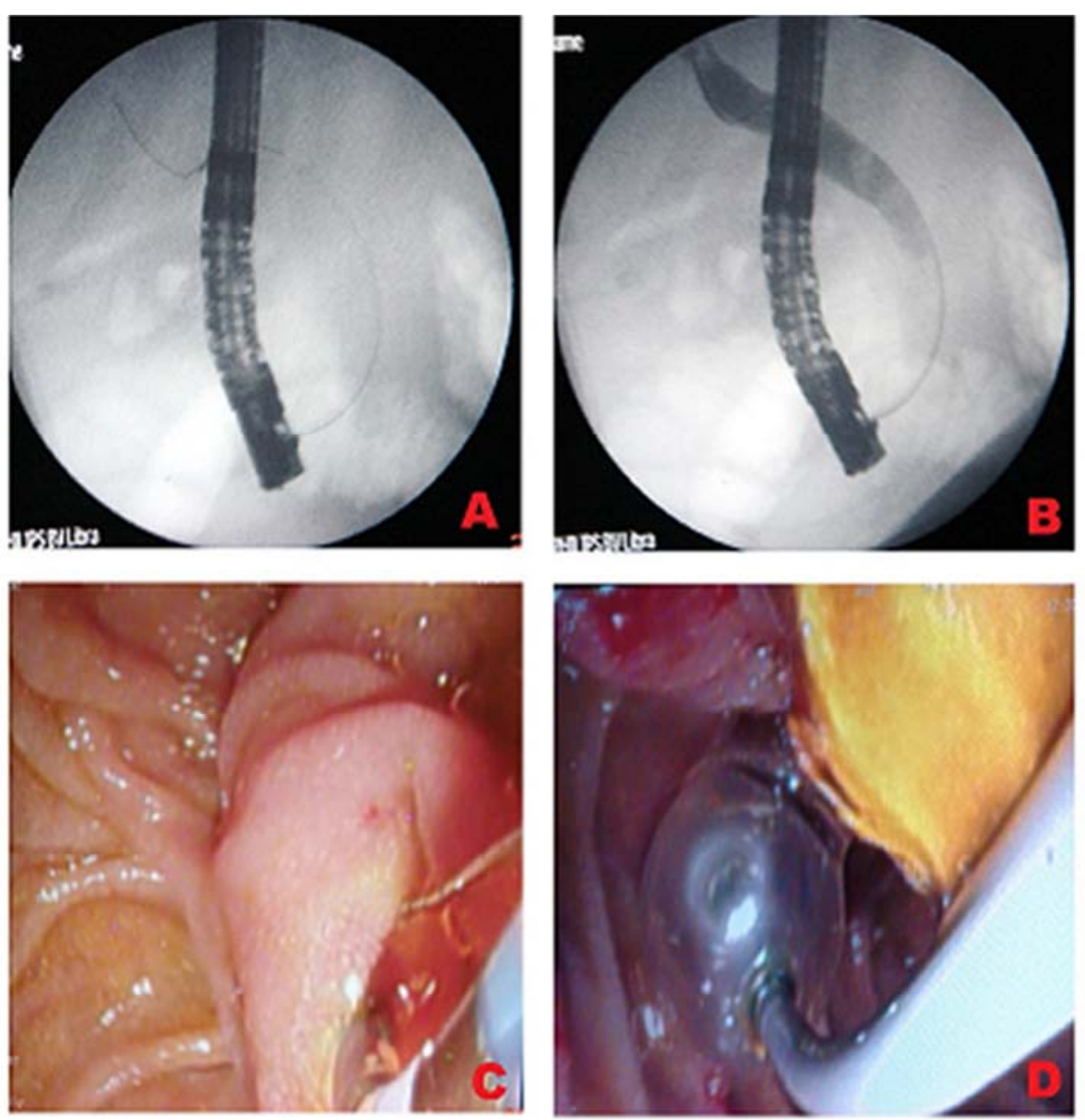

Figure 1. ERCP. A: Endoscopic guide-wire assisted CBD cannulation. B: Contrast injection. C: Endoscopic Sphinctrotomy D: Stone extraction by a balloon catheter.

Anterior longitudinal incision (about $2 \mathrm{~cm}$ ) was done in the lowermost exposed part of the CBD. Through this incision, the 6 Fr. catheter was used to irrigate the CBD with normal saline for several times. This maneuver alone helped most stones go out of the CBD. This was followed by choledochoscopy and balloon sweeping of the CBD stones. When the balloon failed, Dormia basket was used and succeeded to remove all remaining stones. After confirming patency of the CBD by occlusion-cholangiogram (confirming free passage of the contrast into the duodenum with no evident strictures or stones), or, by choledochoscopy, incision was closed with Vicryl 4/0 sutures over a 14 Fr. T-tube.

Normal saline was then injected through the T-tube to confirm patency of the CBD with free flow of saline into the duodenum absence of saline leakage around the T-tube. Finally, a 14 Fr. tubal subhepatic drain was inserted through the right outermost $5 \mathrm{~mm}$ port site, $\mathrm{CO}_{2}$ deflated from the abdomen, trocars removed and skin incisions at trocar sites closed with polyglactic acid $2 / 0$ sutures.

Demographic data, total operative time, CBD clearance success rate, intra- operative and postoperative complications and duration of post-operative hospital stay were recorded for all cases.

Operative time in minutes was define as time between oral advancement of ERCP Duodenoscope in ERCP-LC group, or introduction of camera port in LCBDE-LC group, to, closure of last laparoscopic port.

\subsection{Post-Operative Follow-up}

Postoperatively, patients generally started oral fluids 6 hours after the surgery and progressed as tolerated. Vital signs, subhepatic drain, and T-tube output were recorded regularly.

In group A, patients were discharged after 24 hours provided they are complication free and abdominal $\mathrm{U} / \mathrm{S}$ showed no intra-abdominal collections. In group B, patients were discharged after 48 hours provided they are complication free and abdominal U/S showed no intraabdominal collections. T-tube removal was scheduled on $10^{\text {th }}$ $-14^{\text {th }}$ postoperative day as an outpatient procedure after performing a T-tube cholangiogram.

All patients were clinically examined, had abdominal US and Liver function tests, before their discharge from hospital and then, as outpatients, on post-operative seventh day, fifteenth day, $3^{\text {rd }}$ month, $6^{\text {th }}$ month, and up to 1 year if any symptoms developed.

All patients were discharged on ursodeoxycholic acid 250 mg capsules twice daily for 3 months to decrease incidence of new stones formation or gravels precipitation into the CBD. 

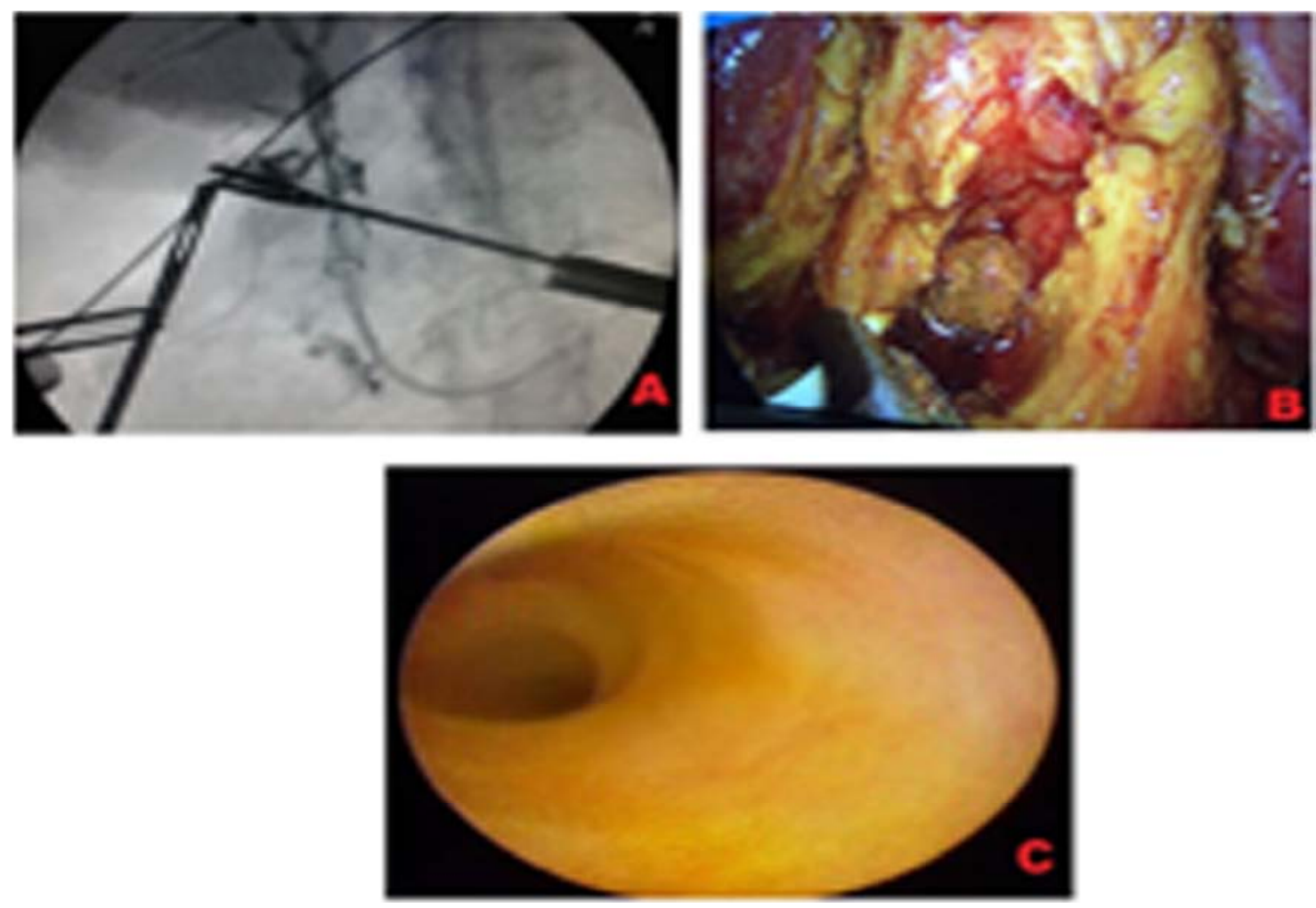

Figure 2. LCBDE. A: Intraoperative cholangiogram. B: Stone extraction by balloon catheter. C: Choledochoscopy.

\subsection{Statistics}

Statistical package SPSS version 20 (SPSS, Chicago, IL, USA) was used to analyze data. Data was presented as numbers, percentages, and arithmetic mean $(\mathrm{M})$ and standard deviation (SD). A $p$-value less than 0.05 was considered significant.

\section{Results}

Patients in this study included 28 females and 18 males with mean age of $42.1 \pm 12.1$ years (range $17-71$ years). Patients were randomly divided into two groups. In group A, patients $(\mathrm{n}=24)$ were treated with single-session intraoperative ERCP (ERCP) and laparoscopic cholecystectomy [ERCP-LC]. In group $\mathrm{B}$, patients $(\mathrm{n}=22)$ were treated with laparoscopic CBD exploration and stone extraction (LCBDE) together with laparoscopic cholecystectomy (LC) [LCBDELC].

No statistically significant difference between the two groups regarding age, gender, mode of presentation, preoperative bilirubin level, multiplicity of $\mathrm{CBD}$ stones, success CBD clearance rate and perioperative complications (table 1 and 2). Group A had statistically significant difference regarding operative time; hospital stay and time of subhepatic drain removal (table 2).

Table 1. Demographic and clinical data of the studied groups.

\begin{tabular}{llll}
\hline Variable & Group (A) [ERCP-LC]. $(\boldsymbol{n}=\mathbf{2 4})$ & Group $(\mathbf{B})$ [LCBD-LC] $(\boldsymbol{n}=\mathbf{2 2})$ & $\boldsymbol{p}$-value \\
\hline Age in years (range) & $44.1 \pm 14.1(17-70)$ & $43 \pm 13.4(19-71)$ & 0.3 \\
female: Male & $15: 9$ & $13: 9$ & 0.526 \\
Abdominal pain & $24(100 \%)$ & $22(100 \%)$ & 1.0 \\
Clinical jaundice & 17 & 15 & 0.549 \\
Serum bilirubin (mg/dL) & $3.5 \pm 2.9$ & $3.6 \pm 3.2$ & 0.34 \\
Single CBD stone & $8(33.4 \%)$ & $7(32 \%)$ & 0.7 \\
Multiple CBD stones & $16(66.6 \%)$ & $15(68 \%)$ & 0.7 \\
\hline
\end{tabular}

Table 2. Comparison between the outcomes of the two groups.

\begin{tabular}{llll}
\hline Variable & Group (A) [ERCP-LC]. $(\boldsymbol{n}=\mathbf{2 4})$ & Group $(\mathbf{B})[\mathbf{L C B D}-\mathbf{L C}](\boldsymbol{n}=\mathbf{2 2})$ & $\boldsymbol{p}$-value \\
\hline Success rate & $22 / 24(91.7 \%)$ & $22 / 22(100 \%)$ & 0.267 \\
Operative Time & $105 \pm 19.1(50-150 \mathrm{~min})$ & $145 \pm 23(100-180 \mathrm{~min})$. & 0.001 \\
Intra-operative complications & 0 & $2 / 22(9 \%)$ & 0.187 \\
Post-operative complications & $3 / 24(12.5 \%)$ & $4 / 22(18 \%)$ & 0.003 \\
Hospital stay & $1.1 \pm 0.91:(1-5$ days $)$. & $2.8 \pm 0.83:(1-6$ days). & 0.000 \\
Drain removal (days) & $2.1 \pm 0.91(2-5)$ & $2.8 \pm 0.83(2-7)$ & \\
\hline
\end{tabular}


Regarding group A, the ERCP successfully cleared the CBD from stones in 22 out of 24 patients (success rate $91.7 \%$ ) at the same same-session and LC completed the treatment. In 2 patients, ERCP failed to extract CBD stones (failed cannulation). The mean operative time was $105 \pm 19.1$ minutes (range 50-150 min.). No intra-operative complications occurred. Early (30 days) post-operative complications occurred in $3 / 24$ patients (12.5\%), 2 patients had mild pancreatitis, and one had postoperative bile leak (less than $50 \mathrm{ml}$ per day) that stopped spontaneously after 4 days. Mean hospital stay was $1.1 \pm 0.91$ days (range 1-5 days).

Regarding group (B), success rate of CBD stone clearance and LC was $100 \%$. Mean operative time was $145 \pm 23$ minutes (range 100-180 min.). Minor intra-operative complications in the form of intra-operative bleeding that was controlled laparoscopicaly occurred in $2 / 22$ cases $(9 \%)$. Minor early post-operative complications occurred in $4 / 22$ patients $(18 \%), 2$ patients had limited bile leak (less than 50 $\mathrm{ml}$ per day) that stopped spontaneously on post-operative days 4 and 5 , one patient had post-operative ileus that resolved on post-operative day 5 , and one patient had postoperative bleeding through the drain that was treated conservatively and stopped spontaneously over 2 hours. Mean hospital stay was 2.8 \pm 0.83 days (range 1-6 days).

During follow-up, none of the patients showed evidence of complications nor missed CBD stones. The mean duration of the postoperative follow-up, for the two groups, was $9 \pm 5.13$ months (range 3-18 months).

\section{Discussion}

Nowadays, options available for the management of choledocholithiasis include ERCP and endoscopic sphincterotomy (pre-operative, intra-operative or postoperative) and common bile duct exploration (laparoscopic or open) [7], However, the extraction of CBD stones laparoscopicaly has gained popularity.

This study was carried out on 46 patients with CBD stones and gallbladder stones, 28 patients $(60.8 \%)$ were females. The most common complaint was abdominal pain in the 46 patients $(100 \%)$, followed by jaundice in 32 patients $(69.5 \%)$. Abdominal ultrasonography and MRCP revealed gallstones in all patients, Single stone in 15 patients (32.6\%) and multiple stones in 31 patients $(67.4 \%)$.

In this study, the procedure was completed in 22 patients $(91.7 \%)$ of group (A); two cases had failed cannulation. Postoperative minor complications occurred in three patients (12.5\%): mild pancreatitis with elevation of serum amylase in two patients and minor bile leak in one patient; no mortality, nearly in agreement with several studies that reported morbidity and mortality rates of $5-11$ and $8-12 \%$, respectively [9].

In group (A), We performed ERCP in left lateral position, but, Williams and Vellacot performed it in the supine position, they found that that the presence of an endotracheal tube in the mouth of the anaesthetized patient neither makes ERCP more difficult, nor does the supine position of the patient [10].

We reported $91.7 \%$ success rate $(22 / 24$ cases $)$, that coincides with those reported by several authors in the literature. Bago et al. reported a success rate $90.2 \%$ in ERCPLC group [11]. Hong et al. compared LC-LCBDE versus ERCP-LC, they reported a success rate of $89.36 \%(126 / 141$ patients) in the LC-LCBDE group and $91.40 \%$ success rate (85/93 patients) in the ERCP-LC group. Cannulation of the CBD failed in six patients of their ERCP-LC group [12]. Williams and Vellacot failed to cannulate the CBD in two of their 13 patients. They stated that despite initial failure to cannulate the $\mathrm{CBD}$ and to deal with the CBD stones, perioperative ERCP allowed the surgeon to see if there were any anatomical problems that would prevent successful postoperative ES if needed in the future [10]. Meyer et al. treated 60 patients with cholecysto-choledocholithiasis by a combined ERCP-LC technique and reported failure to cannulate the papilla in two cases (3\%) [13].

Wright et al. reported a CBD access and stone clearance in all the 14 patients $(100 \%)$ they treated using the ERCP-LC technique [14]. Enochsson et al. using the ERCP-LC could not clear the CBD from stones in 2 out of 31 patients in their series. They performed intraoperative endoscopic sphinctrotomy (ES) and inserted a plastic endoprothesis to secure bile flow until postoperative ERCP was performed successfully [5].

In group (B), the procedure was completed laparoscopicaly in all the 22 cases $(100 \%)$; with no conversion to open surgery. A similar study carried out by Alexakis and Connor denoted a success rate of $80-91 \%$ [15]. In another study by $\mathrm{Lu}$ et al., success rates of $89-95 \%$ were reported [9]. Dasari et al. reported success rate in 72/81 (91\%) patients [16].

Many series reported Successful laparoscopic CBD exploration and clearance in $60-90 \%$ of cases $[8,12,17$, 18].. Berthou et al. found a $97.1 \%$ success rate for laparoscopic common bile duct exploration (LCBDE) followed by choledochotomy [19].

High success rate in this study, (100\%) laparoscopic CBD stone clearance might be due to in part to strict selection criteria especially those for the stones and CBD characters and in part to fixing the working team for all cases. Other studies with large sample size and broader selection criteria are recommended to better test the study hypothesis.

Regarding post-operative complications we agree with Hong et al. who compared ERCP-LC versus LC-LCBDE for the treatment of cholecysto-choledocholithiasis [12. We found no significant differences between both groups with regard to the incidence of postoperative retained stones or complications.

In group (A) Postoperative complications occurred in three patients (12.5\%): minor complications in the form of minimal bile leakage, and two cases of self-limited mild pancreatitis.

In group (B) Minor postoperative complications occurred 
in four patients $(18 \%)$ : in the form of minimal biliary leakage through the subhepatic drain in two patients, post-operative ileus in one patient, and post-operative bleeding in one patient; all of these complications were managed conservatively, and mortality was zero. This was in agreement with several studies that reported a morbidity rate of $8-19 \%$ and a mortality rate of around $0-1 \%$ [15]. This was in contrast to the study carried out by Shojaiefard et al., who reported $5.55 \%$ morbidity but $0 \%$ mortality [20].

The mean operating time in this series has high statistically significant difference between the two groups (105 \pm 19.1 (50-150 min)) vs. (145 $\pm 23(100-180 \mathrm{~min})$.$) , p-vale 0.001)).$ As it is our initial experience, the LCBDE requires a longer learning curve in training for laparoscopic sutures needed in T-tube placement, in comparison with ERCP which was performed with high volume experienced endoscopist with good logistic arrangement. i.e., installation of the endoscopy unit and the C-arm X-ray set. Regarding the significantly less operative time that the ERCP-LC procedure takes and the feasibility to complete the laparoscopic CBD stones extraction in case of ERCP failure, we suggest using this procedure and only if ERCP fails, then LCBDE-LC is referred to at the same session. However, additional larger studies are needed.

Enochsson et al. reported an operating time for ERCP-LC of $192 \pm 8.9 \mathrm{~min}$. They considered the main disadvantage of this procedure to be the prolonged operating time, as in the hands of their surgeons, the mean operating time was prolonged by $85 \mathrm{~min}$, as compared with LC alone, mainly due to logistic factors [5].

Several authors reported a shorter operating time. De Palma et al. reported that the mean duration of the operative time for the combined procedure was $97.7 \pm 30.4 \mathrm{~min}$ (21). Williams and Vellacot reported a median total operating time of $75 \mathrm{~min}$ (50 to $85 \mathrm{~min}$ ) [10]. Meyer et al. reported a mean operating time of $60 \mathrm{~min}$ (40 to $90 \mathrm{~min}$ ) for LC only, and found that the general anesthesia had to be prolonged by 40 min (ranging from 30 to $60 \mathrm{~min}$ ) in order to perform ERCP including the time required for equipment installation [13].

Patients in this study were discharged after a mean hospital stay of $2.1 \pm 0.89$ (1-6 days) days. This is in concordance with the results of other studies in which it ranged from a mean of 2.5 days (ranging from 1 to 5 days) in Williams and Vellacot series [10], $2.6 \pm 0.4$ days (ranging from 1 to 3 days) in Enochsson et al. series [5], and 4.6 days (ranging from 3 to 11 days) in Meyer et al study [13]. In this series, hospital stay was statistically significant longer in LCBDE-LC (1.1 \pm 0 . 91: (1-5 days) vs.2.8 \pm 0.83 : (1-6 days), P value 0.003$)$. Hong et al. has asimilar study reported no statistical difference between two groups regarding hospital stay [12]. In this series, The ERCP group, the patient is managed as if simple laparoscopic cholecystectomy and discharged once passing, while, LCBDE, T-tube care, as well as biliary leak, intra and post-operative bleeding, ileus in some cases, prolonged the hospital stay.

On the other hand, although Berthou et al. had a high success rate in their series of LCBDE-LC, their mean hospital stay was 7.8 days (ranging from 2 to 48 days) [19].
Decker et al. performed LCBDE-LC in 100 patients with primary closure of the CBD without any biliary drainage and reported a median hospital stay of 8 days (ranging from 3 to 32 days) [22].

During the postoperative follow-up period; no patients presented with symptoms, signs, laboratory or US evidence of retained CBDS.

From this study and review of the similar studies, it might be clear that the optimal management of CBDS is dependent on the skills and techniques of the available surgical team and endoscopist. Single-step procedure has some definite advantages over two-step procedure. Both the ERCP-LC and LCBDE-LC techniques can be used effectively and safely for treating cholelithiasis with CBDS, with significantly less operative time and hospital stay in ERCP-LC group, but there is a theoretical presumed issue of post-ES long term sequel of permanent duodenal and pancreatic fluid reflux.

Hospital stay could be kept to a minimum by treating both CBD and gall bladder stones during the same session using the ERCP-LC technique. This method avoids the potential postoperative management problem associated with T-tube that adds to the patient burden and prolongs post-operative stay. Also choledochotomy might increases the risk of biliary leakage and have a potential CBD stricture.

\section{Conclusion}

This study suggests that Single session ERCP-LC and LCBDE-LC procedures for management of cholecystocholedocholithiasis are feasible, safe, and effective and have comparable outcome regarding success rate, peri-operative complications. ERCP-LC has statistically significant less operative time and less hospital stay. However, additional studies with larger sample size and longer follow up are needed.

\section{References}

[1] Sarli L, Pietra N, Franze A, Colla G, Costi R, Gobbi S, et al. Routine intravenous cholangiography, selective endoscopic retrograde cholangiography and endoscopic treatment of common bile duct stones before laparoscopic cholecystectomy. Gastrointest Endosc 1999; 50: 200-8.

[2] Collins C, Maguire D, Ireland A, Fitzgerald E, O'Sullivan GC. A prospective study of common bile duct calculi in patients undergoing laparoscopic cholecystectomy: natural history of choledocholithiasis revisited. Ann Surg 2004; 239: 28-33.

[3] Clayton ES, Connor S, Alexakis N, Leandros E. Meta-analysis of endoscopy and surgery versus surgery alone for common bile duct stones with the gallbladder in situ. Br J Surg. 2006 Oct; 93 (10): 1185-91.

[4] Urbach DR, Khajanchee YS, Jobe BA, Standage BA, Hansen PD, Swanstrom LL. Cost-effective management of common bile duct stones: a decision analysis of the use of endoscopic retrograde cholangiopancreatography (ERCP), intraoperative cholangiography, and laparoscopic bile duct exploration. Surg Endosc 2001; 15: 4-13. 
[5] Enochsson L, Lindberg B, Swahn F, Arnelo U. Intraoperative endoscopic retrograde cholangiopancreatography (ERCP) to remove common bile duct stones during routine laparoscopic cholecystectomy does not prolong hospitalization: a 2-year experience. Surg Endosc 2004; 18: 367-71.

[6] Sherman S, Hawes RH, Rathgaber SW, Uzer MF, Smith MT, Khusro QE, et al. Post-ERCP pancreatitis: randomized, prospective study comparing a low- and high-osmolality contrast agent. Gastrointest Endosc 1994; 40: 422-7.

[7] Rogers S J, Cello J P, Horn J K, Siperstein A E, Schecter W P, Campbell AR, et al. Prospective randomized trial of LC+LCBDE vs ERCP/S+LC for common bile duct stone disease. Arch Surg 2010; 145: 28-33.

[8] Cuschieri A, Croce E, Faggioni A, Jakimowicz J, Lacy A, Lezoche E, et al. EAES ductal stone study. Preliminary findings of multi-center prospective randomized trial comparing two-stage versus single-stage management. Surg Endosc 1996; 10: 1130-5.

[9] Lu J, Cheng Y, Xiong X Z, Lin Y X, Wu S J, Cheng NS. Twostage vssinglestage management for concomitant gallstones and common bile duct stones. World J Gastroenterol 2012; 18: 3156-3166.

[10] Williams GL, Vellacot KD. Selective operative cholangiography and perioperative endoscopic retrograde cholangiopancreatography (ERCP) during laproscopic cholecystectomy. A viable option for choledocholithiasis. Surg Endosc 2002; 16: 465-7.

[11] Bago L, Vicente C, Soler F, Delgado M, Moral I, Guerra I, et al. Two-stage treatment with preoperative endoscopic retrograde cholangiopancreatography (ERCP) compared with single-stage treatment with intraoperative ERCP for patients with symptomatic cholelithiasis with possible choledocholithiasis. Endoscopy 2006; 38: 779-86.

[12] Hong DF, Xin Y, Chen DW. Comparison of laparoscopic cholecystectomy combined with intraoperative endoscopic sphincterotomy and laparoscopic exploration of the common bile duct for cholecystocholedocholithiasis. Surg Endosc 2006; 20: 424-7.

[13] Meyer C, Le Jacques VH, Rohr S, Duclos B, Reimund J,
Baumann R. Management of common bile duct stones in a single operation combining laparoscopic cholecystectomy and perioperative endoscopic sphincterotomy. J Hepatobiliary Pancreat Surg 2002; 9: 196-200.

[14] Wright B, Freeman M, Cumming J, Quickel R, Mandal A. Current management of common bile duct stones: is there a role for laparoscopic cholecystectomy and intraoperative endoscopic retrograde cholangiopancreatography as a single stage procedure? Surgery 2002; 132: 729-37.

[15] Alexakis N, Connor S. Meta-analysis of one- vs. two-stage laparoscopic/ endoscopic management of common bile duct stones. HPB (Oxford) 2012; 14: 254-259.

[16] Dasari B V, Tan C J, Gurusamy K S, Martin D J, Kirk G, Mc Kie L, et al. Surgical versus endoscopic treatment of bile duct stones. Cochrane Database Syst Rev 2013; 12: CD003327.

[17] National Institutes of Health Consensus. Development panel on gallstones and laparoscopic cholecystectomy. J Am Med Assoc 1993; 269: 1018-24.

[18] Rhodes M, Nathanson L, O'Rourke N, Fielding G. Laparoscopic exploration of the common bile duct: lessons learned from 129 consecutive cases. Br J Surg 1995; 82: 6668.

[19] Berthou JC, Drouard F, Charbonneau P, Moussalier K. Evaluation of laparoscopic management of common bile duct stones in 220 patients. Surg Endosc 1998; 12: 16-22.

[20] Shojaiefard A, Esmaeilzadeh M, Ghafouri A, Mehrabi A. Various techniques for the surgical treatment of common bile duct stones: a meta review. Gastroenterol Res Pract 2009; 2009: 840208 .

[21] De Palma GD, Angrisiani L, Lorenzo M, Di Matteo E, Catanzano C, Persico G, et al. Laparoscopic cholecystectomy (LC), intraoperative endoscopic sphincterotomy (ES), and common bile duct stones (CBDS) extraction for management of patients with cholecystocholedocholithiasis. Surg Endosc 1998; 10: 649-52.

[22] Decker G, Borie F, Millat B, Berthou JC, Deleuze A, Drouard $\mathrm{F}$, et al. One hundred laparoscopic choledochotomies with primary closure of the common bile duct. Surg Endosc 2003; 17: $12-8$. 